TRANSACTIONS OF THE

AMERICAN MATHEMATICAL SOCIETY

Volume 351, Number 11, Pages 4585-4597

S 0002-9947(99)02520-9

Article electronically published on July 21, 1999

\title{
A SHARP VERSION OF ZHANG'S THEOREM ON TRUNCATING SEQUENCES OF GRADIENTS
}

\author{
STEFAN MÜLLER
}

\begin{abstract}
Let $K \subset \mathbf{R}^{m n}$ be a compact and convex set of $m \times n$ matrices and let $\left\{u_{j}\right\}$ be a sequence in $W_{\text {loc }}^{1,1}\left(\mathbf{R}^{n} ; \mathbf{R}^{m}\right)$ that converges to $K$ in the mean, i.e. $\int_{\mathbf{R}^{n}} \operatorname{dist}\left(D u_{j}, K\right) \rightarrow 0$. I show that there exists a sequence $v_{j}$ of Lipschitz functions such that $\left\|\operatorname{dist}\left(D v_{j}, K\right)\right\|_{\infty} \rightarrow 0$ and $\mathcal{L}^{n}\left(\left\{u_{j} \neq v_{j}\right\}\right) \rightarrow 0$. This refines a result of Kewei Zhang (Ann. Scuola Norm. Sup. Pisa Cl. Sci. (4) 19 (1992), 313-326), who showed that one may assume $\left\|D v_{j}\right\|_{\infty} \leq C$. Applications to gradient Young measures and to a question of Kinderlehrer and Pedregal (Arch. Rational Mech. Anal. 115 (1991), 329-365) regarding the approximation of $\mathbf{R} \cup\{+\infty\}$ valued quasiconvex functions by finite ones are indicated. A challenging open problem is whether convexity of $K$ can be replaced by quasiconvexity.
\end{abstract}

\section{MAIN RESULTS}

Let $\left\{u_{j}\right\}$ be a sequence of weakly differentiable functions $u_{j}: \mathbf{R}^{n} \rightarrow \mathbf{R}^{m}$ whose gradients approach the ball $B(0, R)$ in the mean, i.e.

$$
\int_{\mathbf{R}^{n}} \operatorname{dist}\left(D u_{j}, B(0, R)\right) d x \rightarrow 0 .
$$

Motivated by work of Acerbi and Fusco [1], [2], and Liu [13], Kewei Zhang showed that the sequence can be modified on a small set in such a way that the new sequence is uniformly Lipschitz. The following theorem is a slight variant of Lemma 3.1 in [21].

Theorem 1 (Zhang). There exists a constant $c(n, m)$ with the following property. If (1.1) holds, then there exists a sequence of functions $v_{j}: \mathbf{R}^{n} \rightarrow \mathbf{R}^{m}$ such that

$$
\left\|D v_{j}\right\|_{\infty} \leq c(n, m) R, \quad \mathcal{L}^{n}\left(\left\{u_{j} \neq v_{j}\right\}\right) \rightarrow 0 .
$$

In fact one has the seemingly stronger conclusions

$$
\mathcal{L}^{n}\left(\left\{u_{j} \neq v_{j} \text { or } D u_{j} \neq D v_{j}\right\}\right) \rightarrow 0, \quad \int_{\mathbf{R}^{n}}\left|D u_{j}-D v_{j}\right| d x \rightarrow 0 .
$$

For the first conclusion it suffices to note that for weakly differentiable functions $u$ and $v$ the implication

$$
u=v \text { a.e. in } A \quad \Longrightarrow \quad D u=D v \text { a.e. in } A
$$

Received by the editors June 23, 1997.

1991 Mathematics Subject Classification. Primary 49J45.

Key words and phrases. Young measures, quasiconvexity, truncation.

(C)1999 American Mathematical Society 
holds (see e.g. [8], Lemma 7.7). For the second conclusion observe that

$$
\left|D u_{j}-D v_{j}\right| \leq\left|D v_{j}\right|+\left|D u_{j}\right| \leq c(n, m) R+R+\operatorname{dist}\left(D u_{j}, B(0, R)\right)
$$

and integrate over the set $\left\{D u_{j} \neq D v_{j}\right\}$.

Theorem 1 has found important applications to the calculus of variations, in particular the study of quasiconvexity, lower semicontinuity, relaxation and gradient Young measures ([9], [21]; see also Corollary 3).

The purpose of the present work is to show that the constant $c(n, m)$ can be chosen arbitrarily close to 1 and that the ball $B(0, R)$ can be replaced by a compact, convex set.

Theorem 2. Let $K$ be a compact, convex set in $\mathbf{R}^{m n}$. Suppose $u_{j} \in W_{\mathrm{loc}}^{1,1}\left(\mathbf{R}^{n}, \mathbf{R}^{m}\right)$ and

$$
\int_{\mathbf{R}^{n}} \operatorname{dist}\left(D u_{j}, K\right) d x \rightarrow 0 .
$$

Then there exists a sequence $v_{j}$ of Lipschitz functions such that

$$
\left\|\operatorname{dist}\left(D v_{j}, K\right)\right\|_{\infty} \rightarrow 0, \quad \mathcal{L}^{n}\left\{u_{j} \neq v_{j}\right\} \rightarrow 0 .
$$

Remarks. 1. A more natural and apparently much harder question is whether the same assertion holds if $K$ is quasiconvex rather than convex.

2. Jan Kristensen pointed out to me that in the scalar case $m=1$ the assumption that $K$ is convex can be dropped. Let $C K$ denote the convex hull of $K$ and $C \operatorname{dist}_{K}$ the convex envelope of the distance function. Kristensen's proof uses (2.14), applied with $C K$ and $(C K)_{\gamma}=C K_{\gamma}$, the identity $C \operatorname{dist}_{K_{\gamma}}=\operatorname{dist}_{(C K)_{\gamma}}$, and the relaxation of nonconvex integral functionals (see e.g. [5]) to obtain

$$
\begin{aligned}
\inf \left\{\int_{B} \operatorname{dist}\left(D v, K_{\gamma}\right) d y: v=u \text { on } \partial B\right\} \\
\quad=\inf \left\{\int_{B} C \operatorname{dist}\left(D w, K_{\gamma}\right): w=u \text { on } \partial B\right\} \\
\quad \leq \int_{B} \operatorname{dist}\left(D \tilde{u},(C K)_{\gamma}\right) \\
\quad \leq\left(1-3^{-n}\right) \int_{B} \operatorname{dist}(D u, C K) d x .
\end{aligned}
$$

A similar argument can be applied for $m>1$ provided that a (somewhat artificial) condition holds which is slightly stronger than the requirement that $C K$ agrees with the quasiconvex hull $Q K$ of $K$.

In the language of Young measures (see [9], [10] for the relevant definitions) one can deduce the following.

Corollary 3. Let $K$ be a compact, convex set in $\mathbf{R}^{m n}$ and let $\Omega \subset \mathbf{R}^{n}$ be open, let $p \geq 1$, and suppose that $\left\{u_{j}\right\}$ generates a $W^{1, p}$ gradient Young measure $\nu=$ $\left\{\nu_{x}\right\}_{x \in \Omega}$ and that

$$
\operatorname{supp} \nu_{x} \subset K \text { for a.e. } x \text { in } \Omega \text {. }
$$


Then there exists a sequence $\left\{v_{j}\right\}$ that generates the same gradient Young measure and satisfies

$$
\left\|\operatorname{dist}\left(D v_{j}, K\right)\right\|_{\infty} \rightarrow 0 .
$$

Warning: There are slightly different definitions of $W^{1,1}$ gradient Young measures in use. Above we have adopted the convention that those measures are generated by sequences for which $\left\{D u_{j}\right\}$ is equi-integrable (and not merely bounded in $L^{1}$ ). No ambiguities arise for $p>1$.

Using Corollary 3, one can simplify the theory of $W^{1, \infty}$ gradient Young measures and answer some of the questions raised in [9] (see Corollary 9 below).

A version of Corollary 3 for Young measures with finite $p$ th moment was discovered by Kristensen [11] and later independently in [7]. It can be used to obtain a simpler approach to $W^{1, p}$ gradient Young measures ([16], [17]).

For $\Omega \neq \mathbf{R}^{n}$, Corollary 3 requires a local version of Theorem 2 .

Theorem 4. Let $K$ be a compact, convex set in $\mathbf{R}^{m n}$, let $\Omega \subset \mathbf{R}^{n}$ be open and let $\left\{u_{j}\right\}$ be a sequence in $W_{\mathrm{loc}}^{1,1}\left(\Omega ; \mathbf{R}^{m}\right)$ that satisfies

$$
\begin{gathered}
u_{j} \rightarrow u_{0} \text { in } L_{\mathrm{loc}}^{1}\left(\Omega ; \mathbf{R}^{m}\right), \\
\operatorname{dist}\left(D u_{j}, K\right) \rightarrow 0 \text { in } L_{\mathrm{loc}}^{1}(\Omega) .
\end{gathered}
$$

Then there exists an increasing sequence of open sets $U_{j}$, compactly contained in $\Omega$, and functions $v_{j} \in W_{\mathrm{loc}}^{1,1}\left(\Omega ; \mathbf{R}^{m}\right)$ such that

$$
\begin{gathered}
v_{j}=u_{0} \text { on } \Omega \backslash U_{j}, \\
\mathcal{L}^{n}\left(\left\{u_{j} \neq v_{j}\right\} \cap U_{j}\right) \rightarrow 0, \\
\left\|\operatorname{dist}\left(D v_{j}, K\right)\right\|_{\infty, \Omega} \rightarrow 0 .
\end{gathered}
$$

Remarks. 1. If $\Omega$ has finite volume, we have $\mathcal{L}^{n}\left(\Omega \backslash U_{j}\right) \rightarrow 0$, and thus $\mathcal{L}^{n}\left(\left\{u_{j} \neq\right.\right.$ $\left.\left.v_{j}\right\}\right) \rightarrow 0$.

2. If $u_{j} \rightarrow u_{0}$ in $W_{\text {loc }}^{1,1}\left(\Omega ; \mathbf{R}^{m}\right)$, then (1.4) holds by the compact Sobolev embedding. In fact, (1.4) and (1.5) imply weak convergence in $W_{\text {loc }}^{1,1}\left(\Omega ; \mathbf{R}^{m}\right)$ (see the proof).

3. Condition (1.6) is a statement of the fact that $u_{0}$ and $v_{j}$ satisfy the same 'boundary condition' (traces may not exist, since we assumed no regularity of $\Omega$ ).

\section{Proofs in $\mathbf{R}^{n}$}

In order to remove the small 'bad' region where $\operatorname{dist}\left(D u_{j}, K\right)>\epsilon$ we locally mollify $u_{j}$. A key point is to use different mollification radii in different regions of $\mathbf{R}^{n}$ (I learned about the use of $x$-dependent mollifiers through the papers [18] and [19] of Schoen and Uhlenbeck). Each mollification step reduces the $L^{1}$ norm of the distance function by a fixed factor, but slightly increases the $L^{\infty}$ norm on the good set. Careful iteration shows, however, that the latter effect can be controlled.

A more precise outline of the proof is as follows. In Lemma 5 we obtain quantitative estimates for mollification on a ball. In Lemma 6 we combine these estimates with a covering argument to achieve the desired reduction of the $L^{1}$ norm. Theorem 7 contains the result of the iteration procedure. Finally, Theorem 2 is an immediate consequence of Theorem 7 . 
In the following $K$ always denotes a compact, convex set in $M:=M^{m \times n}=\mathbf{R}^{m n}$. We use the operator norm $|F|:=\sup \{|F x|:|x|=1\}$ on $M$. The distance function

$$
\operatorname{dist}(A, K)=\min \{|A-F|: F \in K\}
$$

is 1-Lipschitz and convex, since $K$ is convex. Its sublevel sets

$$
K_{\gamma}:=\{A \subset M: \operatorname{dist}(A, K) \leq \gamma\}
$$

are compact and convex, and for $\gamma>0$ and $\delta>0$ one has

$$
\left(K_{\gamma}\right)_{\delta}=K_{\gamma+\delta}, \quad \operatorname{dist}\left(A, K_{\gamma}\right) \leq(\operatorname{dist}(A, K)-\gamma)^{+},
$$

where $a^{+}=\max (a, 0)$. If we let

$$
|K|_{\infty}:=\max \{|A|: A \in K\},
$$

we have

$$
\left|K_{\gamma}\right|_{\infty}=|K|_{\infty}+\gamma, \quad|A| \leq|K|_{\infty}+\operatorname{dist}(A, K) .
$$

By $f_{E} f d x$ we denote the mean value $\left(\mathcal{L}^{n}(E)\right)^{-1} \int_{E} f d x$.

Lemma 5. If $u \in W^{1,1}\left(B(a, r) ; \mathbf{R}^{m}\right)$ and if

$$
\Theta \geq \frac{1}{|K|_{\infty}} f_{B(a, r)} \operatorname{dist}(D u, K) d x, \quad \Theta<8^{-(n+1)}, \quad \gamma:=9 \Theta^{\frac{1}{n+1}}|K|_{\infty},
$$

then there exists $\tilde{u} \in W^{1,1}(B(a, r))$ such that

$$
\begin{gathered}
\tilde{u}=u \quad \text { on } \partial B(a, r), \\
\int_{B(a, r)} \operatorname{dist}\left(D \tilde{u}, K_{\gamma}\right) d x \leq\left(1+\Theta^{\frac{1}{n+1}}\right) \int_{B(a, r) \backslash B(a, r / 2)} \operatorname{dist}(D u, K) d x .
\end{gathered}
$$

Proof. The statement is invariant under the rescaling

$$
u \rightarrow \frac{r}{|K|_{\infty}} u\left(\frac{x-a}{r}\right), \quad K \rightarrow \frac{K}{|K|_{\infty}}, \quad \gamma \rightarrow \frac{\gamma}{|K|_{\infty}} .
$$

We may thus assume $\left|K_{\infty}\right|=1, a=0, r=1$, and we write $B:=B(0,1), B_{\rho}:=$ $B(0, \rho)$. Let $\epsilon \in(0,1 / 8)$ (a specific choice will be made below), and for $x \in B_{7 / 8}$ let

$$
v(x)=\int_{B(x, \epsilon)} u d y=\int_{B_{\epsilon}} u(x+z) d z .
$$

Then

$$
D v(x)=\int_{B(x, \epsilon)} D u d y
$$

and, by convexity of the distance function,

$$
\operatorname{dist}(D v(x), K) \leq \int_{B(x, \epsilon)} \operatorname{dist}(D u, K) d y \leq \epsilon^{-n} \Theta .
$$

Let $\varphi: B \rightarrow[0,1]$ be a cut-off function that satisfies

$$
\varphi \in W_{0}^{1, \infty}\left(B_{7 / 8}\right), \quad \varphi \equiv 1 \text { on } B_{5 / 8}, \quad|D \varphi| \leq 8,
$$


and define

$$
\tilde{u}=(1-\varphi) u+\varphi v .
$$

Then $\tilde{u}=u$ on $B \backslash B_{7 / 8}$ and

$$
D \tilde{u}=(1-\varphi) D u+\varphi D v+(v-u) \otimes D \varphi \text { in } B .
$$

Thus

$$
D \tilde{u}=D u \quad \text { in } B \backslash B_{7 / 8},
$$

We next estimate $v-u$. In view of (2.3) and the assumption $\left|K_{\infty}\right|=1$ we have for a.e. $x \in B_{7 / 8}$

$$
\begin{aligned}
|v-u|(x) & \leq f_{B(x, \epsilon)}|u(y)-u(x)| d y \\
& =\frac{1}{\mathcal{L}^{n}\left(B_{\epsilon}\right)} \int_{0}^{\epsilon} \int_{S^{n-1}}|u(x+\rho e)-u(x)| d \mathcal{H}^{n-1}(e) \rho^{n-1} d \rho \\
& \leq \frac{1}{\mathcal{L}^{n}\left(B_{\epsilon}\right)} \int_{0}^{\epsilon} \int_{S^{n-1}} \int_{0}^{\rho} 1 d t d \mathcal{H}^{n-1}(e) \rho^{n-1} d \rho \\
& +\frac{1}{\mathcal{L}^{n}\left(B_{\epsilon}\right)} \int_{0}^{\epsilon} \int_{S^{n-1}} \int_{0}^{\rho} \operatorname{dist}(D u, K)(x+t e) d t d \mathcal{H}^{n-1}(e) \rho^{n-1} d \rho \\
& =: T_{1}(x, \epsilon)+T_{2}(x, \epsilon) .
\end{aligned}
$$

We have $T_{1}(x, \epsilon)=\epsilon \frac{n}{n+1} \leq \epsilon$, and thus Fubini's theorem yields

$$
\begin{gathered}
\int_{B_{7 / 8} \backslash B_{5 / 8}}(|v-u|-\epsilon) d x \leq \int_{B_{7 / 8} \backslash B_{5 / 8}} T_{2}(x, \epsilon) d x \\
\leq \frac{1}{\mathcal{L}^{n}\left(B_{\epsilon}\right)} \int_{0}^{\epsilon} \int_{S^{n-1}} \int_{0}^{\rho}\left(\int_{B \backslash B_{1 / 2}} \operatorname{dist}(D u, K) d x\right) d t d \mathcal{H}^{n-1}(e) \rho^{n-1} d \rho \\
\leq \epsilon \int_{B \backslash B_{1 / 2}}(\operatorname{dist} D u, K) d x .
\end{gathered}
$$

Since the distance function is convex and 1-Lipschitz, we have

$$
\begin{gathered}
\operatorname{dist}(D \tilde{u}, K) \leq \varphi \operatorname{dist}(D u, K)+(1-\varphi) \operatorname{dist}(D v, K)+|v-u \| D \varphi| \\
\leq \operatorname{dist}(D u, K)+\epsilon^{-n} \Theta+8 \epsilon+8(|v-u|-\epsilon) .
\end{gathered}
$$

Let $\epsilon=\Theta^{\frac{1}{n+1}}$. Then $\epsilon^{-n} \Theta+8 \epsilon=\gamma$ and

$$
\operatorname{dist}(D \tilde{u}, K) \leq \operatorname{dist}(D u, K)+\gamma+8(|v-u|-\epsilon) \quad \text { in } B_{7 / 8} \backslash B_{5 / 8}
$$

The estimate (2.4) gives

$$
\operatorname{dist}(D \tilde{u}, K)<\gamma \quad \text { in } B_{5 / 8} .
$$


Since $D \tilde{u}=D u$ in $B \backslash B_{7 / 8}$, the assertion follows from (2.5), (2.1), and the definition of $\epsilon$.

Lemma 6. There exist positive constants $\alpha(n)<1, c_{2}(n)<1 / 8$, with the following property. If $u \in W_{\mathrm{loc}}^{1,1}\left(\mathbf{R}^{n} ; \mathbf{R}^{m}\right), \gamma \in\left(0,9 c_{2}|K|_{\infty}\right)$ and

$$
\lambda:=\frac{1}{|K|_{\infty}} \int_{\mathbf{R}^{m}} \operatorname{dist}(D u ; K)<\infty,
$$

then there exists a function $\tilde{u} \in W_{\mathrm{loc}}^{1,1}\left(\mathbf{R}^{n} ; \mathbf{R}^{m}\right)$ such that

$$
\begin{gathered}
\frac{1}{|K|_{\infty}} \int_{\mathbf{R}^{n}} \operatorname{dist}\left(D u, K_{\gamma}\right) \leq \alpha(n) \lambda, \\
\mathcal{L}^{n}(\{u \neq \tilde{u}\}) \leq 2^{n}\left(\frac{9|K|_{\infty}}{\gamma}\right)^{n+1} \lambda .
\end{gathered}
$$

Remark. If $D u \in K$ on $R^{n} \backslash V$, then

$$
\begin{gathered}
\{u \neq \tilde{u}\} \subset V_{\rho}=\{x: \operatorname{dist}(x, V) \leq \rho\} ; \\
\rho=c_{7}\left(|K|_{\infty}^{n+1} \gamma^{-(n+1)} \lambda\right)^{1 / n} .
\end{gathered}
$$

Proof. 1. We may suppose $|K|_{\infty}=1$. Let

$$
\begin{aligned}
& \Theta \quad:=\left(\frac{\gamma}{9}\right)^{n+1}<c_{2}^{n+1}<8^{-(n+1)}, \\
& E_{\Theta} \quad:=\left\{x \in \mathbf{R}^{n}: \sup _{r} f_{B(x, r)} \operatorname{dist}(D u, K) d y>\Theta\right\} .
\end{aligned}
$$

Note that by the Lebesgue point theorem

$$
\operatorname{dist}(D u, K) \leq \Theta \quad \text { a.e. in } \mathbf{R}^{n} \backslash E .
$$

Since $c_{2} \leq 1$ we have

$$
\Theta \leq\left(\frac{\gamma}{9}\right)^{n} \gamma \leq \gamma
$$

2. Claim: For each $x \in E_{\Theta}$ there exists a radius $R(x)>0$ such that

$$
\int_{B(x, R(x))} \operatorname{dist}(D u, K) d y \leq \int_{B(x, R(x) / 2)} \operatorname{dist}(D u, K) d y=\Theta .
$$

To prove the claim, consider the function

$$
h(r):=\int_{B(x, r)} \operatorname{dist}(D u, K) d y
$$

and let

$$
R(x)=2 \sup \{r \in(0, \infty): h(r) \geq \Theta\} .
$$

Then $R(x)<\infty$ since $\lambda<\infty$, and $h(R(x) / 2)=\Theta$ by continuity of $h$. The claim is proved.

3. For $R(x)$ as above, consider the family of closed balls

$$
\mathcal{F}=\left\{\overline{B(x, R(x))}: x \in E_{\Theta}\right\} .
$$


By the Besicovitch covering theorem there exist at most $k(n)$ (countable) subfamilies $\mathcal{F}^{(j)}$ of disjoint balls such that the union of the sets

$$
A^{(j)}=\bigcup_{B \in \mathcal{F}^{(j)}} B
$$

covers $E_{\Theta}$. Thus there exists a subfamily $\mathcal{F}^{\prime}$ of disjoint balls such that the set

$$
A=\bigcup_{B \in \mathcal{F}^{\prime}} B
$$

satisfies

$$
\int_{A} \operatorname{dist}(D u, K) d y \geq \frac{1}{k(n)} \int_{E_{\Theta}} \operatorname{dist}(D u, K) d y .
$$

4. In view of (2.10) we may apply Lemma 5 successively to each of the disjoint balls $\overline{B\left(x_{i}, R_{i}\right)} \in \mathcal{F}^{\prime}$ to obtain a function $\tilde{u} \in W_{\text {loc }}^{1,1}\left(\mathbf{R}^{n} ; \mathbf{R}^{m}\right)$ that satisfies

$$
\begin{gathered}
\tilde{u}=u \quad \text { in } \mathbf{R}^{n} \backslash A, \\
\int_{B\left(x_{i}, R_{i}\right)} \operatorname{dist}\left(D \tilde{u}, K_{\gamma}\right) d y \leq\left(1+\Theta^{\frac{1}{n+1}}\right) \int_{B\left(x_{i}, R_{i}\right) \backslash B\left(x_{i}, R_{i} / 2\right)} \operatorname{dist}(D u, K) d y .
\end{gathered}
$$

The definition of $R_{i}=R\left(x_{i}\right)$ (see (2.10)) implies that

$$
\int_{B\left(x_{i}, R_{i} / 2\right)} \operatorname{dist}(D u, K) d y \geq 2^{-n} \int_{B\left(x_{i}, R_{i}\right)} \operatorname{dist}(D u, K) d y .
$$

Hence (2.13) yields

$$
\int_{B\left(x_{i}, R_{i}\right)} \operatorname{dist}\left(D \tilde{u}, K_{\gamma}\right) d y \leq\left(1-2^{-n}\right)\left(1+\Theta^{\frac{1}{n+1}}\right) \int_{B\left(x_{i}, R_{i}\right)} \operatorname{dist}(D u, K) d y .
$$

Let $c_{2}=\min \left(\bar{c}_{2}, 1 / 9\right)$, where $\bar{c}_{2}$ is defined by the equation

$$
\left(1-2^{-n}\right)\left(1+\bar{c}_{2}\right)=\left(1-3^{-n}\right) .
$$

Then the definition of $\Theta$ implies that

$$
\left(1-2^{-n}\right)\left(1+\Theta^{\frac{1}{n+1}}\right) \leq\left(1-3^{-n}\right) .
$$

Since the balls in $\mathcal{F}^{\prime}$ are disjoint and their union is $A$, we deduce that

$$
\int_{A} \operatorname{dist}\left(D \tilde{u}, K_{\gamma}\right) d y \leq\left(1-3^{-n}\right) \int_{A} \operatorname{dist}(D u, K) d y .
$$

On the other hand, (2.12) yields, in combination with (2.8) and (2.9),

$$
\begin{aligned}
& \operatorname{dist}\left(D \tilde{u}, K_{\gamma}\right)=\operatorname{dist}\left(D u, K_{\gamma}\right) \quad \text { in } \mathbf{R}^{n} \backslash A \text {, } \\
& \operatorname{dist}\left(D u, K_{\gamma}\right)=\quad 0 \quad \text { in } \mathbf{R}^{n} \backslash E_{\Theta} .
\end{aligned}
$$

Thus

$$
\int_{\mathbf{R}^{n} \backslash A} \operatorname{dist}\left(D \tilde{u}, K_{\gamma}\right) d y \leq \int_{E_{\Theta} \backslash A} \operatorname{dist}(D u, K) d y .
$$


If we add this to (2.15), use (2.11) and define

$$
\alpha(n)=1-\frac{3^{-n}}{k(n)},
$$

we finally obtain

$$
\int_{\mathbf{R}^{n}} \operatorname{dist}\left(D \tilde{u}, K_{\gamma}\right) d y \leq \alpha(n) \int_{E_{\Theta}} \operatorname{dist}(D u, K) d y .
$$

This proves the first assertion of the lemma.

5. To finish the proof it only remains to estimate $\mathcal{L}^{n}(A)$. One has

$$
\begin{aligned}
\mathcal{L}^{n}(A) & =\sum_{B\left(x_{i}, R_{i}\right) \in \mathcal{F}^{\prime}} \mathcal{L}^{n}\left(B\left(x_{i}, R_{i}\right)\right) \\
& =2^{n} \sum \mathcal{L}^{n}\left(B\left(x_{i}, R_{i} / 2\right)\right) \\
& =2^{n} \sum \frac{1}{\Theta} \int_{B\left(x_{i}, R_{i} / 2\right)} \operatorname{dist}(D u, K) d y \\
& \leq \frac{2^{n}}{\Theta} \lambda=2^{n}\left(\frac{9}{\gamma}\right)^{n+1} \lambda .
\end{aligned}
$$

Hence (2.7) holds, and the lemma is proved.

Proof of the Remark. The function $u$ is only modified on the balls $B\left(x_{i}, R_{i}\right)$, and one has (see point $\mathbf{5}$, above)

$$
\begin{aligned}
\mathcal{L}^{n}\left(B\left(x_{i}, R_{i}\right)\right) & \leq 2^{n} 9^{n+1} \gamma^{-(n+1)} \lambda, \\
2 R_{i} & \leq c_{7}\left(\gamma^{-(n+1)} \lambda\right)^{1 / n}=\rho .
\end{aligned}
$$

Now we must have $x_{i} \in V_{R_{i}} \subset V_{\rho / 2}$ since otherweise $\int_{B\left(x_{i}, R_{i}\right)} \operatorname{dist}(D u, K) d x=0$. Thus $B\left(x_{i}, R_{i}\right) \subset V_{\rho}$.

Let $c_{2}=c_{2}(n)$ be as in Lemma 6 .

Theorem 7. There exists a constant $\bar{c}(n)$ with the following property. Suppose that $K$ is a compact, convex set in $\mathbf{R}^{m n}, u \in W_{\mathrm{loc}}^{1,1}\left(\mathbf{R}^{n}, \mathbf{R}^{m}\right), \gamma \in\left(0,9 c_{2}|K|_{\infty}\right)$ and

$$
\lambda:=\frac{1}{|K|_{\infty}} \int_{\mathbf{R}^{n}} \operatorname{dist}(D u, K) d x<\infty .
$$

Then there exist $v \in W^{1, \infty}\left(\mathbf{R}^{n} ; \mathbf{R}^{m}\right)$ such that

$$
D v \in K_{\gamma} \quad \text { a.e., } \quad \mathcal{L}^{n}\{u \neq v\} \leq \bar{c}(n)|K|_{\infty}^{n+1} \gamma^{-(n+1)} \lambda .
$$

Corollary 8. If $D u \in K$ on $\mathbf{R}^{n} \backslash V$, then

$$
\{u \neq v\} \subset V_{\rho}, \quad \rho=c_{9}\left(|K|_{\infty}^{n+1} \gamma^{-(n+1)} \lambda\right)^{1 / n} .
$$

In fact, values of $u$ outside $V_{\rho}$ play no rôle in the construction of $v$.

Proof of Theorem \%. By scaling we may suppose $|K|_{\infty}=1$. The proof is based on a simple iteration of Lemma 6. Let $\alpha=\alpha(n)$ denote the constant in (2.16) and inductively define

$$
K_{0}=K, \quad K_{i+1}=\left(K_{i}\right)_{\gamma_{i}}, \quad M_{i}=\left|K_{i}\right|_{\infty}
$$




$$
\gamma_{i}=\delta \alpha^{\frac{i}{2(n+1)}} M_{i} .
$$

The value of $\delta>0$ will be chosen below. We have

$$
\ln \frac{M_{i+1}}{M_{i}}=\ln \frac{M_{i}+\gamma_{i}}{M_{i}} \leq \delta \alpha^{\frac{i}{2 n+1}}, \quad M_{0}=1,
$$

and hence

$$
1 \leq M_{i} \leq e^{c_{3} \delta}=: \bar{M}, \quad \sum_{i=0}^{\infty} \gamma_{i} \leq c_{4} \delta c^{c_{3} \delta}=: \bar{\gamma} .
$$

Construct a sequence $u_{i}$ by successive application of Lemma 6 , starting with $u_{0}=u$. Let

$$
\lambda_{i}=\frac{1}{M_{i}} \int_{\mathbf{R}^{n}} \operatorname{dist}\left(D u_{i}, K_{i}\right) d y, \quad \mu_{i}=\mathcal{L}^{n}\left\{u_{i+1} \neq u_{i}\right\} .
$$

By Lemma 6,

$$
\lambda_{i+1} \leq \alpha \lambda_{i}, \quad \mu_{i} \leq 2^{n} 9^{n+1} \bar{M}^{n+1} \gamma_{i}^{-(n+1)} \lambda_{i} .
$$

Thus

$$
\lambda_{i} \leq \alpha^{i} \lambda, \quad \mu_{i} \leq c_{5} \bar{M}^{n+1} \delta^{-(n+1)} \alpha^{i / 2} \lambda,
$$

where $c_{3}, c_{4}$ and $c_{5}$ depend only on the space dimension $n$. Since $\sum \mu_{i}<\infty$, it follows from the definition of $\mu_{i}$ and (1.2) that

$$
u_{i} \rightarrow v, \quad D u_{i} \rightarrow h \text { in measure. }
$$

Moreover,

$$
\int_{\mathbf{R}^{n}} \operatorname{dist}\left(D u_{i}, K_{\bar{\gamma}}\right) \leq \bar{M} \lambda_{i} \rightarrow 0
$$

Application of the dominated convergence theorem with majorant

$$
|K|_{\bar{\gamma}}+\sum_{i} \operatorname{dist}\left(D u_{i}, K_{\bar{\gamma}}\right)
$$

shows that $D u_{i} \rightarrow h$ in $L_{\text {loc }}^{1}\left(\mathbf{R}^{n} ; M\right)$, and by testing with smooth, compactly supported test functions we deduce that

$$
u_{i} \rightarrow v \quad \text { in } W_{\mathrm{loc}}^{1,1}\left(\mathbf{R}^{n} ; \mathbf{R}^{m}\right)
$$

Moreover, by (2.17),

$$
D v \in K_{\bar{\gamma}} \text { a.e. }
$$

and

$$
\mathcal{L}^{n}(\{u \neq v\}) \leq \sum_{i=0}^{\infty} \mu_{i} \leq c_{6} \bar{M}_{i}^{n+1} \delta^{-(n+1)} \lambda .
$$

Now choose $\delta$ such that

$$
\gamma=\bar{\gamma}=c_{4} \delta e^{c_{3} \delta}
$$

Since $\gamma \leq 9 c_{2}$, we have $\delta \leq 9 c_{2} c_{4}^{-1}$ and $\delta \geq \gamma c_{4}^{-1} \exp \left(9 c_{2} c_{3} c_{4}^{-1}\right)$, and now the choice

$$
\bar{c}(n) \leq c_{6} c_{4}^{n+1} \exp \left(18(n+1) \frac{c_{2} c_{3}}{c_{4}}\right)
$$

gives the desired estimate for $\mathcal{L}^{n}(\{u \neq v\})$. 
Proof of Corollary 8. Let $u_{i}$ be as in the proof of Theorem 7, and let

$$
V_{i}=V \cup\left\{u_{i} \neq u\right\}, \quad \rho_{i}=c_{7}\left(\bar{M}^{n+1} \gamma_{i}^{-(n+1)} \lambda_{i}\right)^{1 / n} .
$$

We have $D u_{i} \in K$ in $\mathbf{R}^{n} \backslash V_{i}$, and the remark after Lemma 6 yields

$$
V_{i+1} \subset\left(V_{i}\right)_{\rho_{i}} .
$$

Since $\lambda_{i} \leq \alpha^{i} \lambda$, the definition of $\lambda_{i}$ implies that

$$
\sum \rho_{i} \leq c_{8} \bar{M}^{\frac{n+1}{n}} \delta^{-\frac{n+1}{n}} \lambda^{\frac{1}{n}} .
$$

The assertion now follows from (2.18).

\section{Local estimates}

Proof of Theorem 4. We may suppose $|K|_{\infty}=1$.

1. Claim: $u_{j} \rightarrow u_{0}$ in $W_{\text {loc }}^{1,1}\left(\Omega ; \mathbf{R}^{m}\right), \quad D u_{0} \in K$ a.e.

Proof. Let $U$ be open, $U \subset \subset \Omega$ (as usual, this notion indicates that $\bar{U}$ is compact and contained in $\Omega$ ). For $A \in \mathbf{R}^{m n}$ let $P A$ denote the best approximation of $A$ in the convex, compact set $K$. The sequence $P D u_{j}$ is bounded in $L^{\infty}(U)$, and hence there exists a subsequence that has a weak* limit $h$ in $L^{\infty}(U)$. Since $U$ is bounded, in particular

$$
P D u_{j_{k}} \rightarrow h \text { in } L^{1}(U) .
$$

Now

$$
\left|D u_{j}-P D u_{j}\right|=\operatorname{dist}\left(D u_{j}, K\right) \rightarrow 0 \text { in } L^{1}(U)
$$

and hence $D u_{j_{k}} \rightarrow h$ in $L^{1}(U)$. The usual argument yields $h=D u_{0}$, and uniqueness of the limit implies that the whole sequence converges. Convexity of the distance function and Mazur's and Fatou's lemmas (or standard lower semicontinuity results) show that $\operatorname{dist}\left(D u_{0}, K\right)=0$ a.e. in $U$, and hence a.e. in $\Omega$ by arbitrariness of $U$.

2. Let $V \subset \subset U \subset \subset \Omega$. We construct $v_{j}$ that almost satisfy (1.7) and (1.8). The proof will then be finished by a diagonalization argument. Let $\varphi \in C_{0}^{\infty}(V), 0 \leq$ $\varphi \leq 1$, and define

$$
w_{j}=\varphi u_{j}+(1-\varphi) u_{0} .
$$

Then

$$
D w_{j}=\varphi D u_{j}+(1-\varphi) D u_{0}+\left(u_{j}-u_{0}\right) \otimes D \varphi .
$$

In particular,

$$
\begin{gathered}
D w_{j} \in K \quad \text { in } \Omega \backslash V, \\
\lambda_{j}:=\int_{\Omega} \operatorname{dist}\left(D w_{j}, K\right) d x \leq \int_{V} \operatorname{dist}\left(D u_{j}, K\right) d x+\int_{V}\left|u_{j}-u_{0}\right||D \varphi| d x .
\end{gathered}
$$

By the assumptions, $\lambda_{j} \rightarrow 0$. Let $\delta>0$. In view of Theorem 6 and Corollary 8 there exists $j_{0}=j_{0}(U, V, \varphi, \delta)$ such that for all $j \geq j_{0}$ there exist $v_{j} \in W_{\text {loc }}^{1,1}\left(\Omega, \mathbf{R}^{m}\right)$ that satisfy

$$
\left\{v_{j} \neq w_{j}\right\} \subset U, \quad \mathcal{L}^{n}\left(v_{j} \neq w_{j}\right)<\delta, \quad D v_{j} \in K_{\delta} \text { a.e. }
$$

It follows that

$$
v_{j}=u_{0} \quad \text { in } \Omega \backslash U
$$




$$
\begin{aligned}
\mathcal{L}^{n}\left(\left\{v_{j} \neq u_{j}\right\} \cap U\right) & <\delta+\mathcal{L}^{n}(\{\varphi \neq 1\} \cap V)+\mathcal{L}^{n}(U \backslash V), \\
\operatorname{dist}\left(D v_{j}, K\right) & \leq \delta .
\end{aligned}
$$

3. Let $\left\{\tilde{U}_{k}\right\}$ be an increasing sequence of open sets $\tilde{U}_{k} \subset \subset \Omega$ whose union exhausts $\Omega$. Let $V_{k} \subset \subset \tilde{U}_{k}$ and $\varphi_{k} \in C_{0}^{\infty}\left(V_{k}\right)$ be such that

$$
\mathcal{L}^{n}\left(\tilde{U}_{k} \backslash V_{k}\right)<\frac{1}{k}, \quad \mathcal{L}^{n}\left(\left\{\varphi_{k} \neq 1\right\} \cap V\right)<\frac{1}{k},
$$

$0 \leq \varphi_{k} \leq 1$, and let $\delta_{k}<\frac{1}{k}$. By point 2 , there exists $j_{k}$ such that for $j \geq j_{k}$ there exist functions $v_{j}$ that satisfy

$$
\begin{gathered}
v_{j}=u_{0} \quad \text { in } \Omega \backslash \tilde{U}_{k}, \\
\left.\mathcal{L}^{n}\left(\left\{v_{j} \neq u_{j}\right\}\right) \cap \tilde{U}_{k}\right)<\frac{3}{k}, \quad \operatorname{dist}\left(D v_{j}, K\right)<\frac{1}{k} .
\end{gathered}
$$

We may suppose without loss of generality that $j_{k}$ is (strictly) increasing. To finish the proof, define

$$
U_{j}=\tilde{U}_{k} \quad \text { if } j_{k} \leq j<j_{k}+1
$$

\section{Applichtion to quasiconvex functions}

A function $f$ from the $m \times n$ matrices $\mathbf{R}^{m n}$ to $\mathbf{R} \cup\{-\infty, \infty\}$ is called quasiconvex if for all bounded domains $U \subset \mathbf{R}^{n}$ with $\mathcal{L}^{n}(\partial U)=0$ and all $F \in \mathbf{R}^{m n}$

$$
\int_{U} f(F+D \eta) d x \geq \int_{U} f(F) d x=\mathcal{L}^{n}(U) f(F) \quad \forall \eta \in W_{0}^{1, \infty}\left(U ; \mathbf{R}^{m}\right),
$$

whenever the integral on the left exists.

Quasiconvexity is the fundamental notion in the vector-valued calculus of variations (see [14], [15], [3], [4], [6], [20]). It states that affine functions minimize the functional $u \mapsto \int_{U} f(D u)$ subject to their own boundary conditions. Quasiconvexity is difficult to handle, however, since no local characterization is known for $n, m>1$ (and cannot exist for $m \geq 3, n \geq 2$; see [12]). Even the approximation of general quasiconvex functions by finite ones is a largely open question. As a corollary of Theorem 2 we obtain at least the following result, which answers the question in [9], p. 350, equation (5.19) (see pp. 342 and 345 for the relevant definitions). We remark that every $\mathbf{R}$-valued quasiconvex function is continuous and even locally Lipschitz, since it is rank-1 convex (see e.g. [4]).

Corollary 9. Let $K \subset \mathbf{R}^{m n}$ be a convex, compact set with non-empty interior. Let $f: \mathbf{R}^{m n} \rightarrow \mathbf{R} \cup\{-\infty, \infty\}$ be a quasiconvex function that satisfies

$$
f \in C(K ; \mathbf{R}), \quad f=+\infty \text { on } \mathbf{R}^{m n} \backslash K .
$$

Then, for all $F \in K$,

$$
f(F)=\sup \left\{g(F) \mid g: \mathbf{R}^{m n} \rightarrow \mathbf{R}, g \leq f \text { on } K, g \text { quasiconvex }\right\} .
$$

Proof. 1. We may assume $0 \in \operatorname{int} K$, since quasiconvexity is invariant under translation in $\mathbf{R}^{m n}$. We have

$$
K \subset \lambda \text { int } K, \quad \forall \lambda>1 .
$$


Indeed, if $A \in \partial K$, then $t A+(1-t) B \in K$ for all $t \in(0,1)$ and all $B$ in a small neighbourhood of 0 . Hence $t A \in \operatorname{int} K$, for all $t \in(0,1)$. Thus (4.2) holds.

2. Let $G_{\infty}$ denote the right hand side of (4.1) and let $P$ denote the nearest neighbour projection onto $K$. For $k \in \mathbf{N} \cup\{0\}$ define

$$
h_{k}(F)=f(P F)+k \operatorname{dist}(F, K) \leq f(F) .
$$

Let $g_{k}=h_{k}^{q c}$ denote the quasiconvex hull of $h_{k}$, i.e. the largest quasiconvex function below $h_{k}$. Thus $g_{k}(F) \leq G_{\infty}$. On the other hand, by standard relaxation results (see e.g. [4], Chapter 5, Theorem 1.1)

$$
g_{k}(F)=\inf \left\{\int_{Q} h_{k}(D u) d x: u-F x \in W_{0}^{1, \infty}\left(Q, \mathbf{R}^{m}\right)\right\},
$$

where $Q=(0,1)^{n}$. Hence there exist Lipschitz functions $u_{k}$ such that

$$
\limsup _{k \rightarrow \infty} \int_{Q} h_{k}\left(D u_{k}\right) d x \leq G_{\infty}, \quad u_{k}=F x \text { on } \partial Q .
$$

In particular,

$$
\int_{Q} \operatorname{dist}\left(D u_{k}, K\right) \rightarrow 0
$$

Hence $D u_{k}$ is bounded in $L^{1}$, and after possible passage to a subsequence we may assume that $u_{k} \rightarrow u_{0}$ in $L^{1}$.

3. By Theorem 4 there exist $v_{k} \in W^{1, \infty}\left(Q, \mathbf{R}^{m}\right)$ which satisfy

$$
\begin{gathered}
\mathcal{L}^{n}\left(\left\{u_{k} \neq v_{k}\right\}\right) \rightarrow 0, \quad v_{k}=F x \text { on } \partial Q, \\
\left\|\operatorname{dist}\left(D v_{k}, K\right)\right\|_{\infty} \rightarrow 0 .
\end{gathered}
$$

Taking into account (1.2), the uniform continuity of $h_{0}$ and the inequality $h_{0} \leq h_{k}$, we see that

$$
\limsup _{k \rightarrow \infty} \int_{Q} h_{0}\left(D v_{k}\right) d x=\limsup _{k \rightarrow \infty} \int_{Q} h_{0}\left(D u_{k}\right) d x \leq G_{\infty} .
$$

In view of (4.2) and (4.5) there exist $\lambda_{k} \searrow 1$ such that $\lambda_{k}^{-1} D v_{k} \in K, \lambda_{k}^{-1} F \in K$. Using the uniform continuity of $h_{0}$ as well as quasiconvexity and continuity of $f$, we obtain

$$
\begin{aligned}
f(F) & =\lim _{k \rightarrow \infty} f\left(\lambda_{k}^{-1} F\right) \leq \limsup _{k \rightarrow \infty} \int_{Q} f\left(\lambda_{k}^{-1} D v_{k}\right) d x \\
& =\limsup _{k \rightarrow \infty} \int_{Q} h_{0}\left(\lambda_{k}^{-1} D v_{k}\right) d x \leq G_{\infty} .
\end{aligned}
$$

The proof is finished.

\section{ACKNOWLEDGEMEnts}

It is a pleasure to thank G. Alberti, J. Kristensen and V. Šverák for very interesting discussions. 


\section{REFERENCES}

1. E. Acerbi and N. Fusco, Semincontinuity problems in the calculus of variations, Arch. Rat. Mech. Anal. 86 (1984), 125-145. MR 85m:49021

2. E. Acerbi and N. Fusco, An approximation lemma for $W^{1, p}$ functions, in: Material instabilities in continuum mechanics and related mathematical problems (J.M. Ball, ed.), Oxford UP, 1988, pp. 1-5. MR 89m:46060

3. J.M. Ball, Convexity conditions and existence theorems in nonlinear elasticity, Arch. Rat. Mech. Anal. 63 (1977), 337-403. MR 57:14788

4. B. Dacorogna, Direct methods in the calculus of variations, Springer, 1989. MR 90e:49001

5. I. Ekeland and R. Temam, Convex analysis and variational problems, North Holland, Amsterdam, 1976. MR 57:3931b

6. L.C. Evans, Weak convergence methods for nonlinear partial differential equations, CBMS no. 74, 1990, Amer. Math. Soc. MR 91a:35009

7. I. Fonseca, S. Müller and P. Pedregal, Analysis of concentration and oscillation effects generated by gradients, SIAM J. Math. Anal. 29 (1998), 736-756. CMP 98:11

8. D. Gilbarg and N.S. Trudinger, Elliptic partial differential equations of second order, Springer, 2nd ed., 1983. MR 86c:35035

9. D. Kinderlehrer and P. Pedregal, Characterization of Young measures generated by gradients, Arch. Rat. Mech. Anal. 115 (1991), 329-365. MR 92k:49089

10. D. Kinderlehrer and P. Pedregal, Gradient Young measure generated by sequences in Sobolev spaces, J. Geom. Analysis 4 (1994), 59-90. MR 95f:49059

11. J. Kristensen, Finite functionals and Young measures generated by gradients of Sobolev functions, Ph.D. Thesis, Technical University of Denmark, Lyngby.

12. J. Kristensen, On the non-locality of quasiconvexity, Ann. Inst. H. Poincaré Anal. Non Linéaire 16 (1999), 1-13.

13. F.-C. Liu, A Luzin type property of Sobolev functions, Indiana Univ. Math. J. 26 (1977), 645-651. MR 56:8782

14. C.B. Morrey, Quasi-convexity and the lower semicontinuity of multiple integrals, Pacific J. Math. 2 (1952), 25-53. MR 14:992a

15. C.B. Morrey, Multiple integrals in the calculus of variations, Springer, 1966. MR 34:2380

16. P. Pedregal, Parametrized measures and variational principles, Birkhäuser, 1997. MR 98e: 49001

17. M. Sychev, A new approach to Young measure theory, relaxation and convergence in energy, to appear in Ann. Inst. H. Poincaré Anal. Non Linéaire.

18. R. Schoen and K. Uhlenbeck, A regularity theory for harmonic maps, J. Differ. Geom. 17 (1982), 307-335; 18 (1983), 329. MR 84b:58037

19. R. Schoen and K. Uhlenbeck, Boundary regularity and the Dirichlet problem for harmonic maps, J. Differ. Geom. 18 (1983), 253-268. MR 85b:58037

20. V. Šverák, Lower semicontinuity of variational integrals and compensated compactness, in: Proc. ICM 1994, vol. 2, Birkhäuser, 1995, pp. 1153-1158. MR 97h:49021

21. K. Zhang, A construction of quasiconvex functions with linear growth at infinity, Ann. Scuola Norm. Sup. Pisa 19 (1992), 313-326. MR 94d:49018

Max Planck Institute for Mathematics in the Sciences, Inselstr. 22-26, 04103 Leipzig, Germany

E-mail address: sm@mis.mpg.de 